\title{
Numerical Simulation and Clinical Implications of Stenosis in Coronary Blood Flow
}

\author{
Jun-Mei Zhang, ${ }^{1}$ Liang Zhong, ${ }^{1,2}$ Tong Luo, ${ }^{3}$ Yunlong Huo, ${ }^{4}$ Swee Yaw Tan, ${ }^{1,2}$ \\ Aaron Sung Lung Wong, ${ }^{1,2}$ Boyang Su, ${ }^{1}$ Min Wan, ${ }^{1}$ Xiaodan Zhao, ${ }^{1}$ Ghassan S. Kassab, ${ }^{3}$ \\ Heow Pueh Lee, ${ }^{5}$ Boo Cheong Khoo, ${ }^{5}$ Chang-Wei Kang, ${ }^{6}$ Te Ba, ${ }^{6}$ and Ru San Tan ${ }^{1,2}$ \\ ${ }^{1}$ National Heart Center Singapore, 5 Hospital Drive, Singapore 169609 \\ ${ }^{2}$ Duke-NUS Graduate Medical School Singapore, 8 College Road, Singapore 169857 \\ ${ }^{3}$ Department of Biomedical Engineering, Indiana University-Purdue University Indianapolis, Indianapolis, IN 46202, USA \\ ${ }^{4}$ Department of Mechanics and Engineering Science, College of Engineering, Peking University, Beijing 100871, China \\ ${ }^{5}$ Department of Mechanical Engineering, National University of Singapore, 1 Engineering Drive 2, Singapore 117576 \\ ${ }^{6}$ Fluid Dynamics Department, Institute of High Performance Computing, 1 Fusionopolis Way, \\ No. 16-16 Connexis North, Singapore 138632 \\ Correspondence should be addressed to Liang Zhong; zhong.liang@nhcs.com.sg
}

Received 28 February 2014; Accepted 29 April 2014; Published 2 June 2014

Academic Editor: Bruno Levy

Copyright (C) 2014 Jun-Mei Zhang et al. This is an open access article distributed under the Creative Commons Attribution License, which permits unrestricted use, distribution, and reproduction in any medium, provided the original work is properly cited.

Fractional flow reserve (FFR) is the gold standard to guide coronary interventions. However it can only be obtained via invasive angiography. The objective of this study is to propose a noninvasive method to determine $\mathrm{FFR}_{\mathrm{CT}}$ by combining computed tomography angiographic (CTA) images and computational fluid dynamics (CFD) technique. Utilizing the method, this study explored the effects of diameter stenosis (DS), stenosis length, and location on $\mathrm{FFR}_{\mathrm{CT}}$. The baseline left anterior descending (LAD) model was reconstructed from CTA of a healthy porcine heart. A series of models were created by adding an idealized stenosis (with DS from $45 \%$ to $75 \%$, stenosis length from $4 \mathrm{~mm}$ to $16 \mathrm{~mm}$, and at 4 locations separately). Through numerical simulations, it was found that $\mathrm{FFR}_{\mathrm{CT}}$ decreased (from 0.89 to 0.74 ), when DS increased (from $45 \%$ to $75 \%$ ). Similarly, $\mathrm{FFR}_{\mathrm{CT}}$ decreased with the increase of stenosis length and the stenosis located at proximal position had lower $\mathrm{FFR}_{\mathrm{CT}}$ than that at distal position. These findings

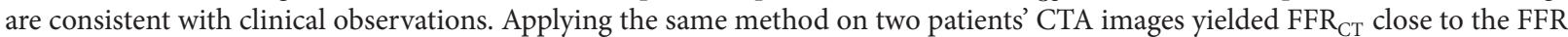
values obtained via invasive angiography. The proposed noninvasive computation of $\mathrm{FFR}_{\mathrm{CT}}$ is promising for clinical diagnosis of CAD.

\section{Introduction}

As the most common type of cardiovascular disease, coronary artery disease (CAD) is caused by the build-up of plaques on the endothelial walls of coronary arteries, which leads to a reduction in arteries cross-sectional area and blood supply to the myocardium [1]. Pathology studies of CAD have revealed that coronary stenosis is the predominant factor leading to cardiovascular-related events such as myocardial infarction (MI), stroke, and unstable angina. Therefore it is vital to physiologically characterize and quantify functional coronary stenosis.
The rapid development of noninvasive imaging technologies, such as computed tomography angiography (CTA) and magnetic resonance imaging (MRI), has proven valuable to characterize the anatomic severity of CAD with fair cost and less complication. Diameter stenosis (DS) is commonly applied to quantify the anatomic severity of CAD. It expresses the ratio of the lumen diameter at a stenotic region over that of a "normal" segment. However, DS cannot characterize hemodynamic functional significance of coronary stenosis on myocardial blood supply. Among the stenoses discriminated with the threshold of $50 \%$ for DS, fewer than half are ischemic [2]. 
In terms of hemodynamic indexes to quantify the functional significance of coronary stenosis, fractional flow reserve (FFR) is the gold standard to guide coronary interventions [3-6] because of its significantly fewer follow-up coronary events over DS. FFR is calculated as a ratio of the pressure distal versus pressure proximal to a stenosis following vasodilation. Revascularization is commonly recommended when the coronary stenosis leads to FFR $\leq 0.80$. However, FFR can only be measured via invasive coronary catheterization, which may result in higher medical cost and complications [4]. This is not ideal, because only one-third of the intermediate stenosis (DS $=40-70 \%$ ) is classified as functional significant with FFR $\leq 0.80$ [5].

In view of the abundant hemodynamic information provided by computational fluid dynamics (CFD) simulations, considerable insights have been gained on understanding the physiology of CAD through CFD studies [7]. Tremendous progress has been made in applying the CFD method to simulate the blood flow in patient-specific coronary artery models for elucidating the role of hemodynamics in CAD development and progression [8-10].

As proper boundary conditions are necessary for CFD simulations of CAD, both prescribed profiles [11, 12] and sophisticated reduced-ordered models [13-15] have been attempted. For the latter, the downstream vasculature is represented by flow-dependent formulation. For example, the static pressure at the outlet may be calculated from the corresponding flow rate and resistance. Since resistance, compliance, or impedance values of the downstream vasculature are assumed to be decided by their own anatomy rather than by the upstream stenosis, these parameters obtained from the normal vessel (without stenosis) can be applied to the diseased vessel [15].

With the development of CFD techniques, numerous CFD studies have been coupled with clinical imaging techniques to provide detailed hemodynamic information and link CAD with vortices [16], wall shear stress (WSS) [12, 17], oscillatory shear index (OSI) [18], and so forth. However, the application of CFD method to predict clinical diagnosis indexes is still limited. A landmark study was carried out by HeartFlow Company [19-22] to derive noninvasive $\mathrm{FFR}_{\mathrm{CT}}$ from CT images by applying sophisticated reduced-ordered models as the boundary conditions for transient numerical simulations. Because the calculation of FFR is based on the time-averaged pressure over several cardiac cycles in clinics [23], this study explored a method to combine CT images and steady flow simulation for calculating noninvasive FFR $_{\mathrm{CT}}$ with lower computational cost. The method is firstly applied on a left anterior descending (LAD) model reconstructed from CTA of a healthy porcine heart and then tested on two patient-specific left coronary artery trees reconstructed from patients' CTA images to examine whether the approach can be applied for noninvasively assessing the hemodynamic significance of coronary stenosis in clinics.

As the anatomical severity of CAD is believed to be related not only to DS but also to the location and length of the stenosis, which can affect the hemodynamics [24], a series of models were created by adding an idealized stenosis (with DS from $45 \%$ to $75 \%$, stenosis length from $4 \mathrm{~mm}$ to $16 \mathrm{~mm}$, and at 4 locations separately) on the baseline porcine LAD model. In this manner, the effects of DS, stenosis length, and location (proximal to distal) on $\mathrm{FFR}_{\mathrm{CT}}$ are investigated. The results may be useful to aid the clinician in making the decision of revascularization.

This paper is organized as follows: the detailed computational methodologies were described in Section 2. In Section 3, the detailed hemodynamic information (flow patterns, flow rate, pressure, and $\mathrm{FFR}_{\mathrm{CT}}$ distributions) for a series of stenosed porcine LAD models was provided to explore the effect of DS, stenosis length, and location on $\mathrm{FFR}_{\mathrm{CT}}$. The results of two case studies were also presented to demonstrate the applicability of the current methodologies on clinical diagnosis of human CAD. Finally, the conclusions were drawn in Section 4.

\section{Computational Methodologies}

In this study, a left anterior descending (LAD) model was used as the baseline model, which was reconstructed from CTA of a healthy porcine heart. The detailed description on animal preparation, CTA, and image processing can be found in [25]. As shown in Figure 1(a), the baseline model embodied the LAD main trunk ( $\geq 2 \mathrm{~mm}$ in diameter) and 7 primary branches ("a"-"g" in Figure 1) ( $\geq 1 \mathrm{~mm}$ in diameter). To investigate the effect of DS, the trunk between side branches "a" and "b" was narrowed to represent stenosed model with DS of $45 \%, 55 \%, 65 \%$, and $75 \%$ separately as shown in Figure 2. All of these stenoses were located at the same location (Location A) with the same stenosis length of $4 \mathrm{~mm}$. To investigate the effect of stenosis location, a $4 \mathrm{~mm}$ length stenosis with DS of $55 \%$ was created at 4 locations (Location "A" to "D") from proximal to distal LAD trunk, as shown in Figure 3. Another series of models were constructed with a $55 \%$ stenosis located downstream the branch "a" and having the stenosis lengths of $4 \mathrm{~mm}, 8 \mathrm{~mm}, 12 \mathrm{~mm}$, and $16 \mathrm{~mm}$ separately, as shown in Figure 4.

After generating these LAD models with SpaceClaim, their computational domains were discretized with commercial software ANSYS workbench. Meshes near the branch junctions, at the stenosis and near the walls, were refined for adequate resolution of flow in the boundary layers (as shown in Figure 1(b)). After mesh dependency test conducted on the baseline model, a total of about 0.5 million volume cells were found to be adequate, as further grid refinement led to less than $1 \%$ relative error in the maximum velocity. The same setting for mesh generation was applied for all other LAD models.

In order to simulate the blood flow in normal and diseased LAD models, the continuity (1) and Navier-Stokes (2) equations were solved by FLUENT using finite volume approximation:

$$
\begin{gathered}
\frac{\partial u_{j}}{\partial x_{j}}=0 \\
\frac{\partial\left(\rho u_{j} u_{i}\right)}{\partial x_{j}}=-\frac{\partial P}{\partial x_{i}}+\frac{\partial}{\partial x_{j}}\left(\mu\left(\frac{\partial u_{i}}{\partial x_{j}}+\frac{\partial u_{j}}{\partial x_{i}}\right)\right),
\end{gathered}
$$




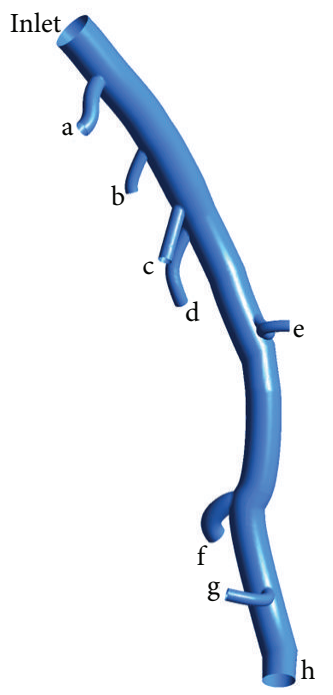

(a)

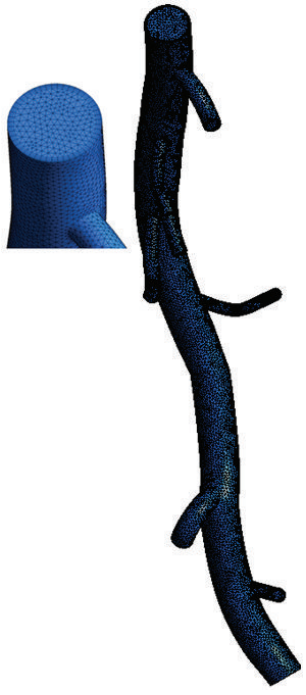

(b)

FIgURE 1: (a) Baseline LAD model reconstructed from CT and (b) the generated meshes for the baseline model with enlarged view.

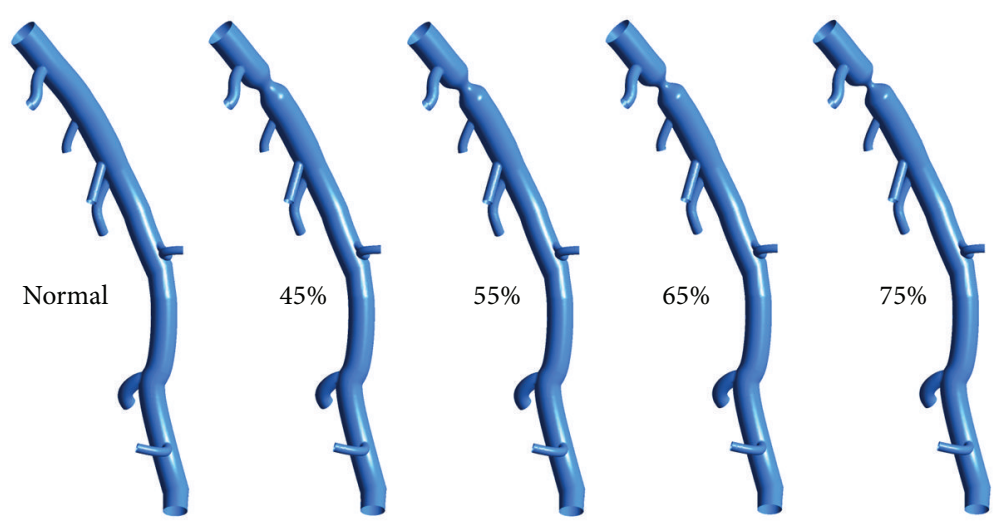

FIGURE 2: Baseline and stenosed LAD models with the $4 \mathrm{~mm}$ length stenoses located at the same location (Location A) with DS of $45 \%$, $55 \%$, $65 \%$, and $75 \%$ separately.

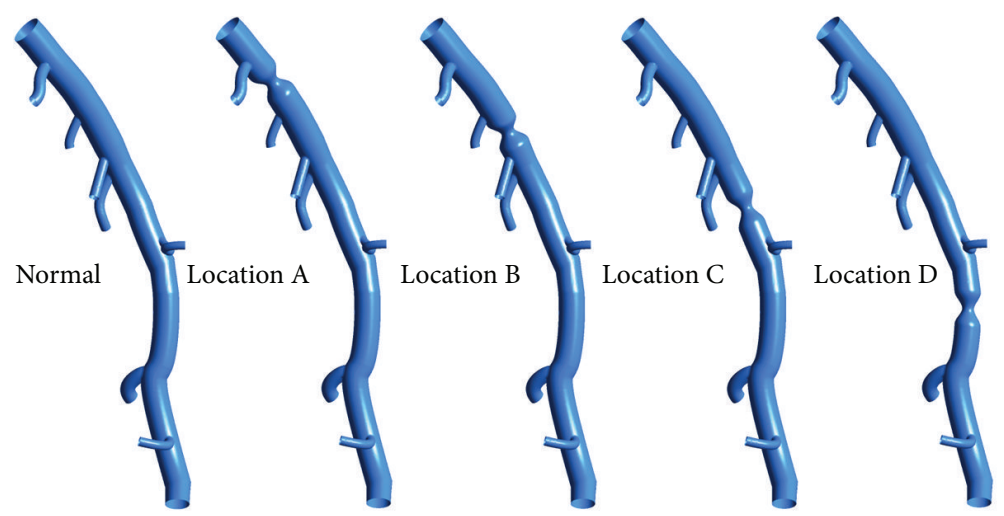

FIGURE 3: Baseline and stenosed LAD models with the $4 \mathrm{~mm}$ length stenoses located at A, B, C, and D separately (having the DS of 55\%). 


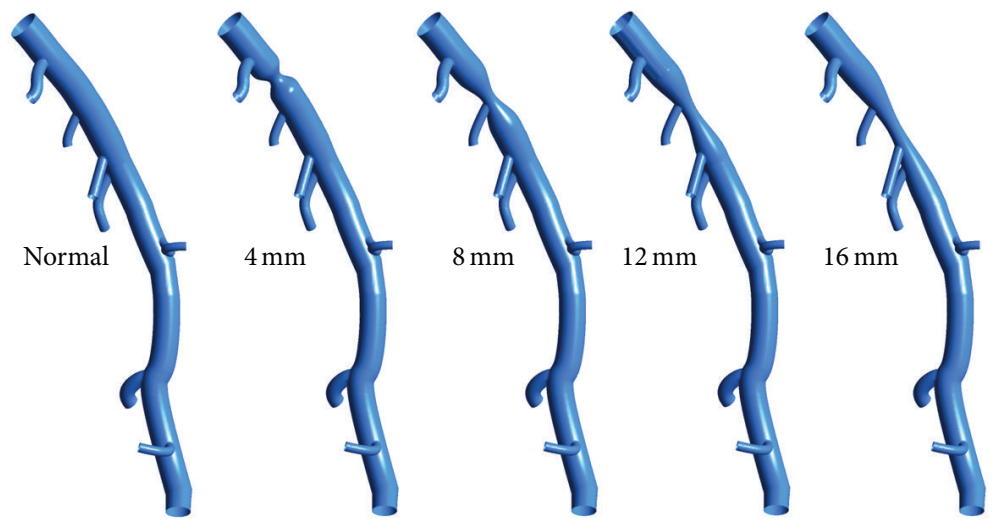

FIGURE 4: Baseline and stenosed LAD models with 55\% DS located downstream the branch "a" with the stenosis lengths of $4 \mathrm{~mm}, 8 \mathrm{~mm}$, $12 \mathrm{~mm}$, and $16 \mathrm{~mm}$ separately.

Here $x_{j}$ and $u_{j}\left(\right.$ or $\left.u_{i}\right)$ were the location in Cartesian coordinate and the Cartesian component of velocity, respectively. $P$ represented the static pressure. $\rho$ and $\mu$ were density and dynamic viscosity of the fluid, which were set as $1060 \mathrm{~kg} / \mathrm{m}^{3}$ and $4.5 \times 10^{-3} \mathrm{~Pa} \cdot \mathrm{s}$, respectively, to mimic the blood properties in large epicardial arteries [25, 26].

Proper boundary conditions are required for CFD simulations to closely mimic the real physiological condition [13]. In general, the total pressure was specified at the inlet of each model, and resistance boundary condition was assigned to every coronary outlet to enforce a relationship between pressure and flow.

According to the pulsatile pressure and flow velocity waveforms measured in vivo at the inlet of the porcine LAD [25], the time-averaged inflow rate and pressure were $66.75 \mathrm{~mL} / \mathrm{min}$ and $69.54 \mathrm{mmHg}$, respectively.

In order to derive the resistance values of downstream vasculatures, the steady-state simulations under two sets of boundary conditions were carried out on the baseline model prior to the simulation on stenosed LAD models. One of the simulations represents the peak phase of systole, when the inlet pressure is at the maximum [25]. The other simulation represents the peak diastole phase with minimum pressure at the inlet. For both simulations, the flow rate distributions between the primary branches were believed to obey the generalized Murray's law [27], as it was the baseline LAD, which represents normal (healthy) coronary arteries without stenosis. Based on these two steady-state flow simulations, the pressure and flow rate information at the outlets of primary branches could be obtained. Accordingly the resistance of the vasculature downstream each primary branch was obtained via

$$
R_{i}=\frac{P_{i}-P_{0, i}}{Q_{i}},
$$

where $P_{i}$ and $Q_{i}$ represent the pressure and flow rate at the $i$ th outlet, respectively. Here, $R_{i}$ is the resistance of the downstream vasculature and $P_{0, i}$ is the back pressure at $i$ th outlet.
In clinics, FFR measurement is done following vasodilation, which leads to the decrease of coronary resistance. To resemble this physiological situation, the resistance of downstream vasculature of each primary branch was assumed to be reduced to 0.21 times of its resting value, which was within the physiological range measured by Wilson et al. [24]. Accordingly the total pressure at the inlet was assumed to be $69.87 \mathrm{mmHg}$ for all the porcine LAD models with the consideration of flow rate increment following vasodilation. Because the epicardial stenosis severity was found to be not affecting the minimal microvascular resistance [28], the resistance of downstream vascular for a stenosed LAD model was assumed to be the same as that of the baseline model [22].

User defined function (UDF) was compiled to supply the resistance boundary conditions at the outlets. In this manner, the downstream vasculature of the primary branches was coupled to the LAD model, and the static pressure at each outlet was solved iteratively. To ensure smooth convergence, the pressure gradient calculated at the outlet was attenuated several times to couple iterative underrelaxationbased resistance boundary conditions with those outlets; namely, an implicit algorithm was applied to the outlet with an underrelaxation factor of $\omega$ given as

$$
P_{i}^{n+1}=P_{i}^{n}+\omega\left(R_{i} Q_{i}^{n+1}+P_{o, i}-P_{i}^{n}\right) .
$$

In addition, no-slip boundary condition was applied at the wall, as all these LAD models were assumed to be stationary and rigid. All the computations were executed in a Dell T7500 workstation and it took around half hour computational time for one case.

\section{Results and Discussion}

To elucidate the role of stenosis on hemodynamics of coronary artery, Figure 5 shows the streamline distributions in the normal and stenosed LAD models with DS increasing from $45 \%$ to $75 \%$ at interval of $10 \%$. When the LAD was free of stenosis, part of the inflow diverted in the primary branches along its way downstream. Most of the blood flowed through 

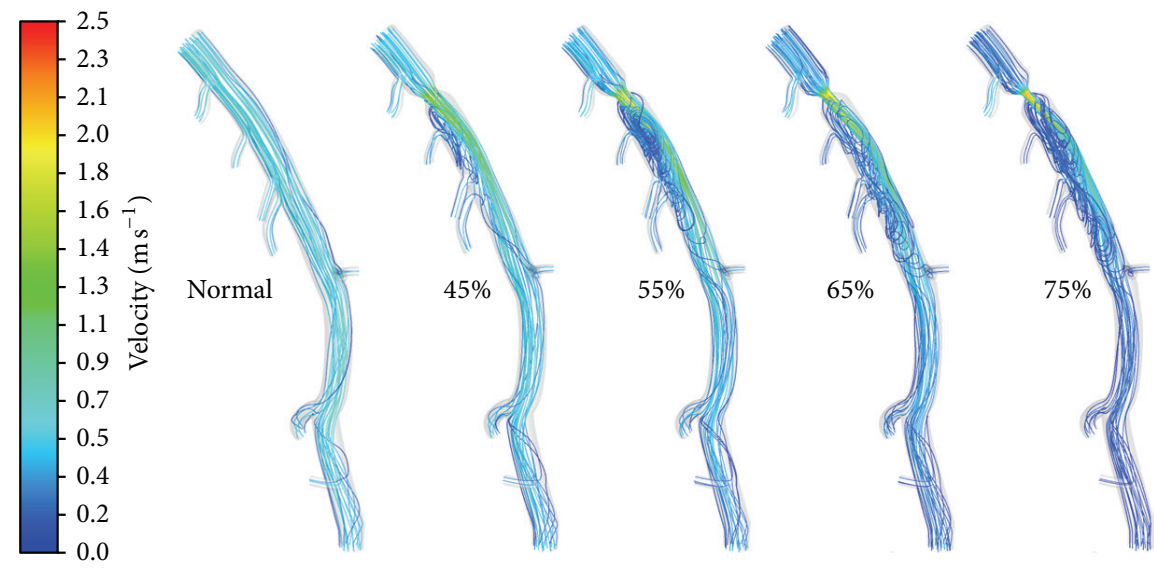

FIGURE 5: Streamline distributions on the baseline and stenosed LAD models with the $4 \mathrm{~mm}$ length stenoses located at the same location (Location A) with DS of $45 \%, 55 \%, 65 \%$, and $75 \%$ separately.

the LAD main trunk smoothly, with streamlines parallel to the walls. In other words, there was no obvious presence of vortex in the normal LAD model.

Once there was a stenosis in the LAD trunk, the streamlines diverted into the center of the LAD main trunk due to the shrinking of the cross-sectional area from the beginning to the throat of the stenosis region. Downstream the throat of the stenosis, the cross-sectional area of the LAD main trunk increased gradually. Due to the inertia momentum, the fluid moved downstream with slight divergence of the streamlines. Hence flow recirculation region was formed near the wall downstream the stenosis, which was filled by vortices and reverse flow derived from the downstream streamlines as shown in Figure 5. With the increase of DS, the throat diameter became smaller leading to more significant divergence of the streamlines and larger flow recirculation in size downstream the stenosis. These vortices and flow recirculation were suspected to promote thrombus formation and potentially myocardial infarction [16].

An interesting phenomenon was found for the flow rate distribution among the primary branches. With the increase of DS, less blood flowed downstream to perfuse the myocardium downstream the first primary branch (labeled as "a" in Figure 1). This is because, for this series of models, the stenosis was located at the main trunk downstream the first primary branch. The vortices and flow recirculation developed downstream the stenosis result in energy diffusion and dissipation, which makes it difficult to perfuse the blood downstream. Hence less blood was distributed to the regions downstream the stenosis. However, there was a slight increase of blood flow to the first primary branch, with the increase of DS as shown in Figure 6. Similar phenomenon is named as "branch steal" by Gould et al. [29] to describe the situation when a nonstenotic branch between proximal and distal stenoses shunts flow away from the stenotic parallel daughter branch to an extent depending on their relative size and severities of the 2 stenoses, which leads to less flow in the stenotic daughter branch. It was also found that the sum of

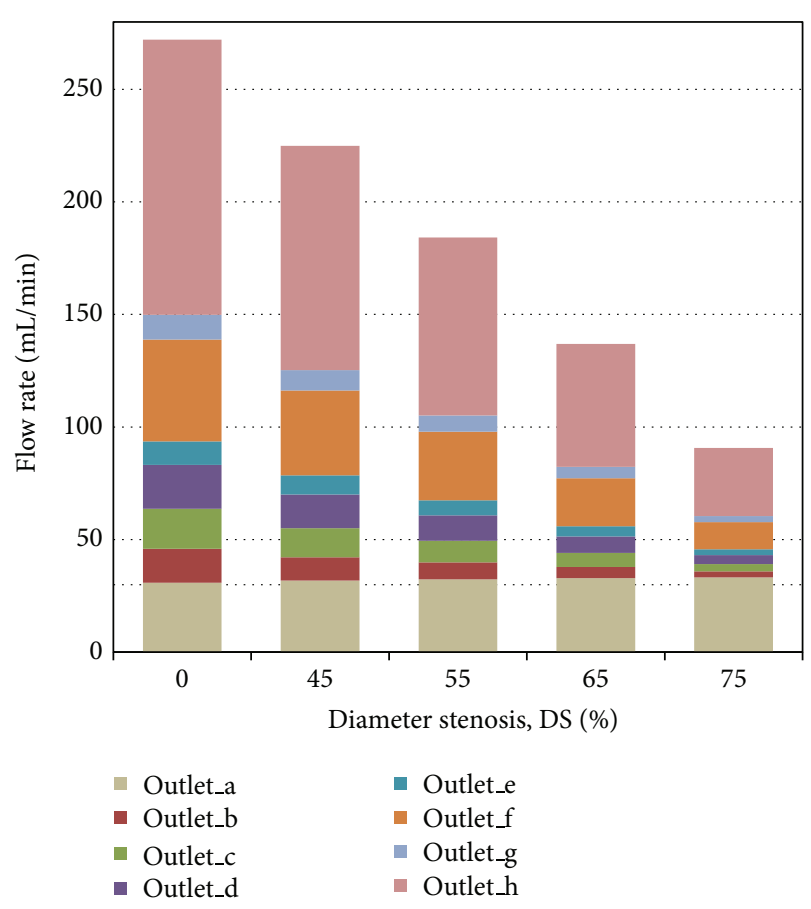

FIGURE 6: Flow rate distributions for the baseline and stenosed LAD models with the $4 \mathrm{~mm}$ length stenoses located at the same location (Location A) with DS of $45 \%, 55 \%, 65 \%$, and $75 \%$ separately.

the total blood flow through the outlets reduced with the increase of DS, which implied that stenosis led to a reduction in blood supply to the myocardium. This was the most important physiological complication of coronary stenosis.

To quantify the hemodynamic significance of stenosis, Figure 7 shows the pressure distribution for different models. For normal LAD, the pressure drop along the main trunk was mainly due to the viscous force, which was less than $3.3 \mathrm{mmHg}$. Due to the diffusion and dissipation of 

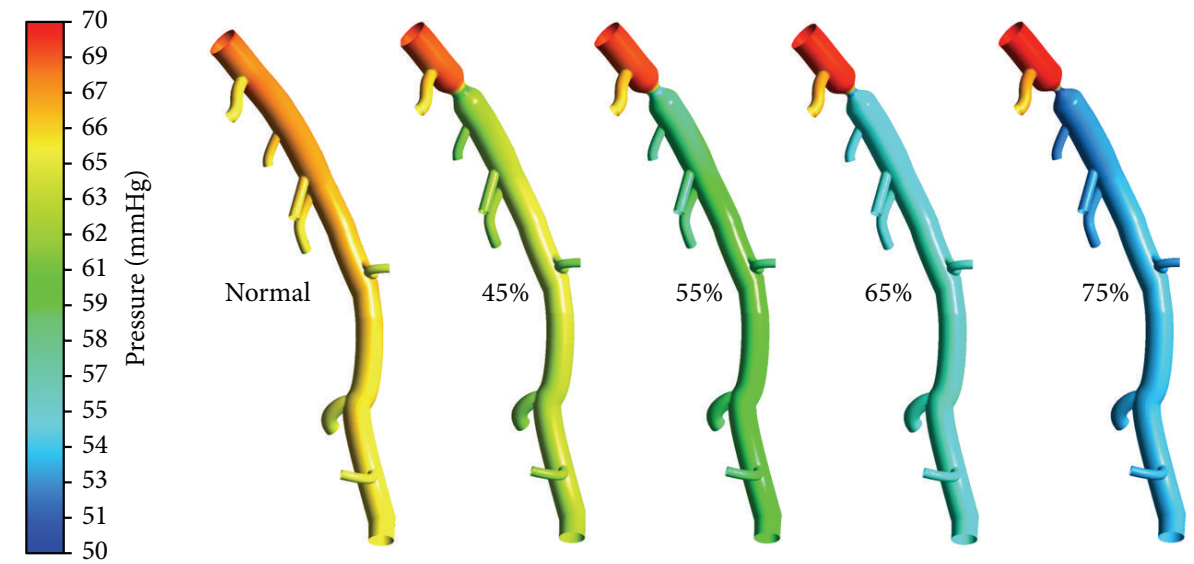

FIGURE 7: Pressure distributions on the baseline and stenosed LAD models with the $4 \mathrm{~mm}$ length stenoses located at the same location (Location A) with DS of $45 \%, 55 \%, 65 \%$, and $75 \%$ separately.

the recirculation vortices formed downstream the stenosis, the pressure therein decreased much more. With the increase of DS, pressure downstream the stenosis became much lower as shown in Figure 7.

To resemble FFR values measured via invasive angiography, $\mathrm{FFR}_{\mathrm{CT}}$ is calculated as $P_{d} / P_{a}$. Here $P_{d}$ and $P_{a}$ represent the pressure distal and proximal to the stenosis, respectively. As shown in Figure 8(a), FFR $_{\mathrm{CT}}$ decreased from 0.89 to 0.74 , when DS increased from $45 \%$ to $75 \%$. As revascularization is commonly recommended when coronary stenosis leads to FFR $\leq 0.8$ in clinical applications [5], the LAD with DS $\geq$ $60 \%$ was suggested to receive revascularization procedures according to Figure 8(a). This is in line with the clinical findings that the diagnostic performance of CTA can be improved by rating coronary stenosis as significant with the threshold of $60 \%$ rather than $50 \%$ [30].

To assess the effect of stenosis location and length on the hemodynamic severity of CAD, Figures 8(b) and 8(c) show the relationship between stenosis location and length with $\mathrm{FFR}_{\mathrm{CT}}$, respectively. Comparing with the stenosis located more distally, those stenoses located at proximal portion led to lower $P_{d} / P_{a}$, namely, $\mathrm{FFR}_{\mathrm{CT}}$. Therefore the stenosis located at proximal portion resulted in more significant reduction in blood supply to the myocardium, which was suspected to promote the further accumulation of plaques. This result is consistent with the clinical findings that the adverse events of coronary artery disease occurred most frequently in a proximal position [31].

In addition, the $\mathrm{FFR}_{\mathrm{CT}}$ values decreased with the increase of stenosis length, which implies higher fractional losses over the stenosis. However the effect of lesion length on hemodynamics is not significant as shown in Figure 8(c). Similar finding was reported by Wilson et al. [24]. However it is worth noting that fractional losses at longer stenosis may depend on the ruggedness of the surface and local geometry of the stenosis in real life. Hence simulation on the realistic coronary artery tree is critically important.
Therefore, we have also attempted to apply the same CFD methodologies on the patients' CTA images. The detailed information on image reconstruction and numerical simulation was presented in $[32,33]$. Figures $9(a)$ and $9(\mathrm{~b})$ show the predicted $\mathrm{FFR}_{\mathrm{CT}}$ on the patient-specific left coronary artery trees reconstructed from two patients' CTA images, respectively. The first patient has a moderate stenosis at proximal LAD, which is not ischemia-causing, as its FFR was 0.97 measured via invasive angiography. Its $\mathrm{FFR}_{\mathrm{CT}}$ predicted by CFD was 0.98 . The other patient has an ischemia-causing moderate stenosis at mid LAD, whose FFR was less than 0.8 , that is, at 0.73 , and its $\mathrm{FFF}_{\mathrm{CT}}$ was found to be 0.74 . These two examples demonstrated that the noninvasive methodologies presented in this study were applicable to assess the hemodynamic significance of stenosis in clinics.

\section{Conclusions}

In recent years, tremendous progress has been made on invasive and noninvasive medical imaging techniques to diagnose the anatomical and hemodynamic significance of coronary stenosis. Although FFR is the gold standard to diagnose the hemodynamic significance of the CAD, it can only be obtained via invasive coronary angiography. Combining CTA with CFD methods, the flow pattern and pressure distributions can be predicted for a baseline LAD model reconstructed from the CTA of a healthy porcine heart.

By adding stenosis on this baseline model, the effects of DS, stenosis location, and length on the hemodynamics of stenosed coronary artery were explored. It was found that the flow recirculation vortices were formed downstream the throat of stenosis due to the divergence of the streamline, which led to the decrease of flow rate and pressure downstream the stenosis due to energy diffusion and dissipation. The phenomenon of "branch steal" was observed, which was previously reported by Gould et al. [29] when a nonstenotic 


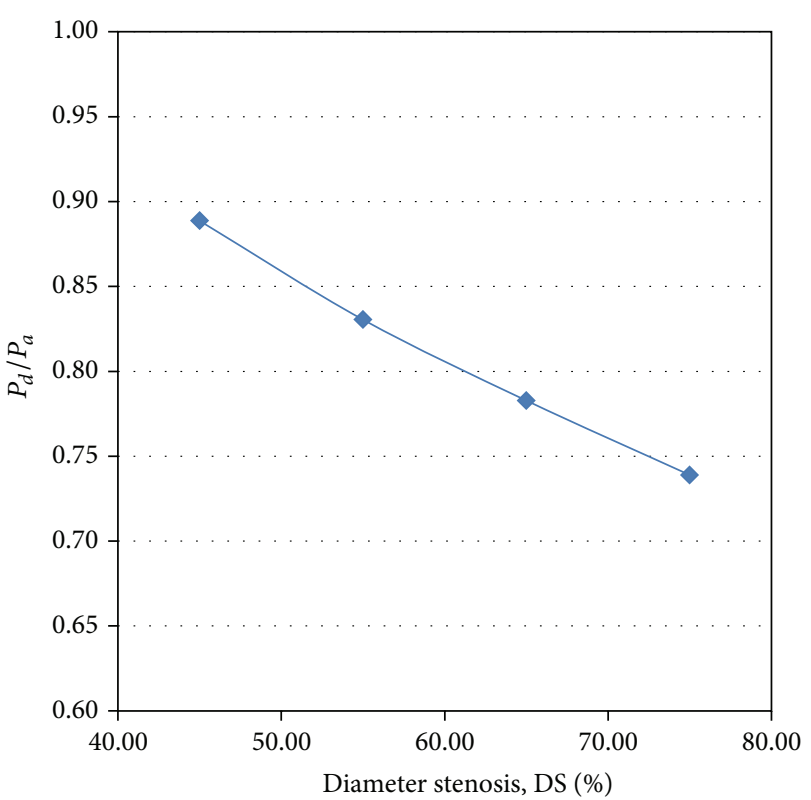

(a)

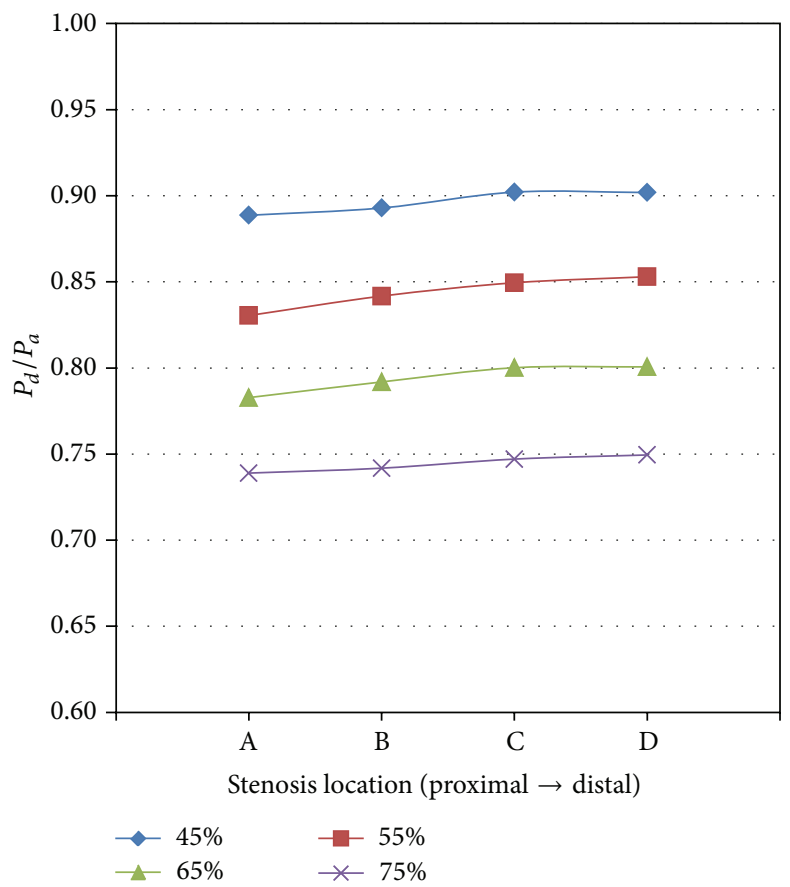

(b)

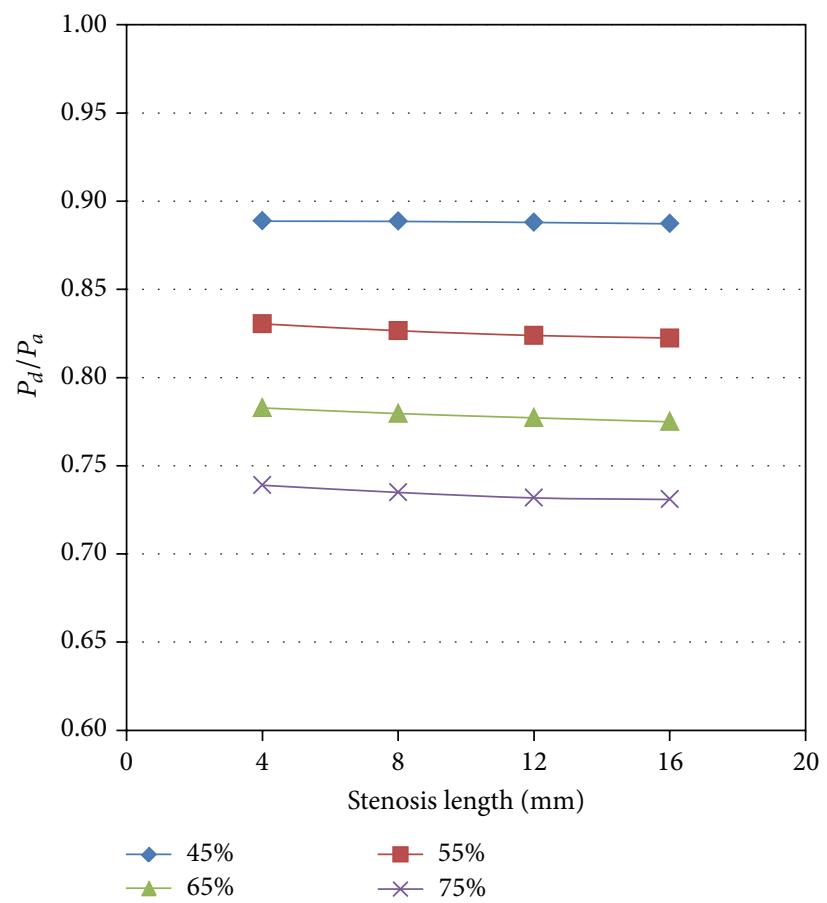

(c)

FIGURE 8: Effect of (a) diameter stenosis, (b) stenosis location, and (c) length on $\mathrm{FFR}_{\mathrm{CT}}$.

branch between proximal and distal stenoses shunts flow away from the stenotic parallel daughter branch [28].

With the increase of DS, the flow recirculation region increased in size, which led to lower pressure and $\mathrm{FFR}_{\mathrm{CT}}$ downstream stenosis. Using a threshold of 0.8 , the LAD with DS $\geq 60 \%$ was suggested to receive revascularization procedure rather than $\mathrm{DS} \geq 50 \%$, which is in line with the clinical finding [30].

In addition, the stenosis location and length were found to affect the hemodynamics in coronary artery trees. 


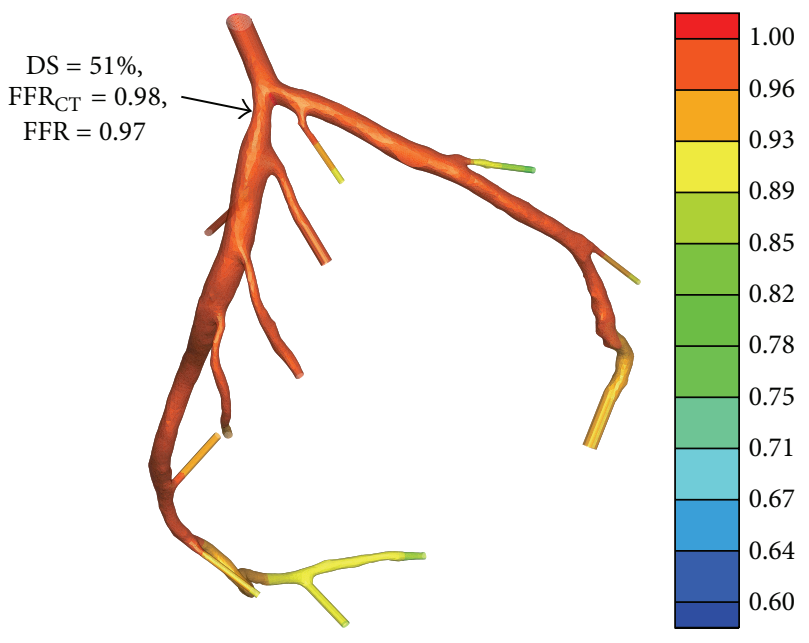

(a)

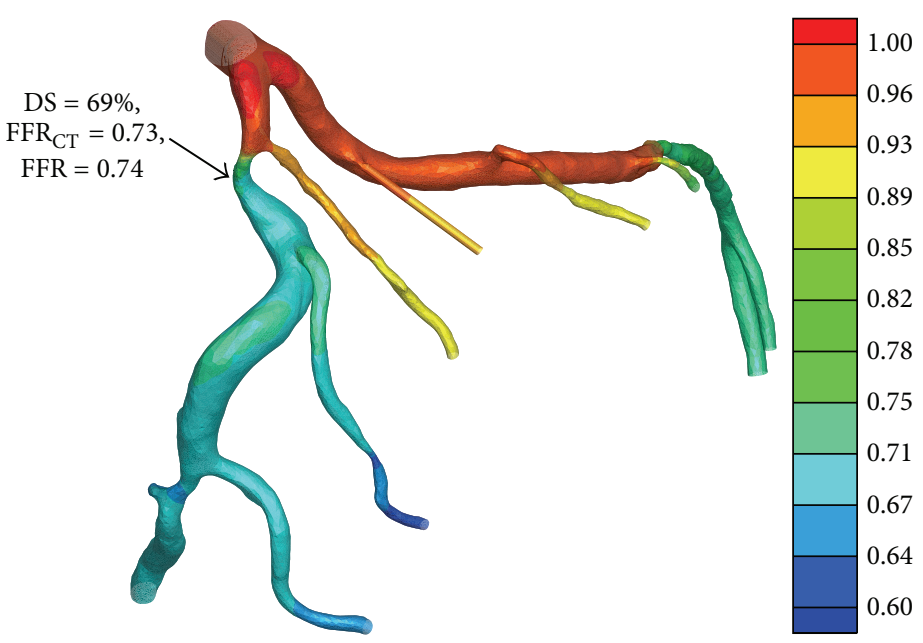

(b)

FIGURE 9: Distributions of $\mathrm{FFR}_{\mathrm{CT}}$ based on numerical simulations on patient-specific left coronary trees reconstructed from two patients' CTA images. (a) The patient has a moderate stenosis $\left(\mathrm{DS}=51 \%\right.$ ) at proximal $\mathrm{LAD}$, whose $\mathrm{FFR}$ is 0.97 via invasive angiography and its $\mathrm{FFR} \mathrm{CT}_{\mathrm{C}}$ is 0.98 predicted by CFD. (b) The patient has a moderate stenosis ( $\mathrm{DS}=69 \%$ ) at mid LAD, whose FFR is 0.73 via invasive angiography and its $\mathrm{FFR}_{\mathrm{CT}}$ is 0.74 predicted by CFD.

Lower $\mathrm{FFR}_{\mathrm{CT}}$ was associated with the stenosis located in the proximal position and/or with longer stenosis length. This was in line with the clinical observations [24, 31].

Further application of the methodologies on two patientspecific left coronary artery trees reconstructed from CTA images demonstrated that the methodology utilized in this study was promising in facilitating the patient-specific noninvasive diagnosis of hemodynamic significance of coronary stenosis with affordable computational cost. Currently, the computation time was half hour for one case study with a Dell T7500 workstation, which is much longer than an invasive clinical setting, but it is acceptable for noninvasive diagnostics. If a more powerful workstation with larger number of compute nodes is applied, the computation time can be much less than 30 minutes.

Although efforts have been made to mimic the physiological condition as close as possible, there are several possible sources of limitation inherent in the assumptions made in the present study. First, the determination of downstream vascular resistance is argumentative. Intracoronary resistance has been reported to be fluctuating in a phasic pattern, even after administration of vasodilation medicine [23, 34, 35], due to the interaction between the myocardium and microvasculature during systole and diastole. However a mean resistance value was estimated (by iterative approximation at two time points) in this study. To justify the effect of resistance value on $\mathrm{FFR}_{\mathrm{CT}}$, a series of simulations were carried out on one model. It was found that the $24-71 \%$ variations of resistance values only led to less than $2.4 \%$ differences of $\mathrm{FFR}_{\mathrm{CT}}$. Therefore the error in the estimated downstream vascular resistance may only result in limited effect on the results of $\mathrm{FFR}_{\mathrm{CT}}$. In addition, the method in this study does not consider the circumference with microvascular disease, which is an interesting topic to be explored in the future.
Secondly, the compliance effect of the vessel walls was ignored in this study, as compliance was reported to be less important in affecting the hemodynamics of the coronary arteries [36]. Thirdly, the blood flow was assumed to be laminar with Newtonian fluid. Since the vessels investigated in this study had a diameter greater than $1 \mathrm{~mm}$ and the blood flow in the elderly was generally correlated with the low hematocrit, the assumption of Newtonian fluid can be justified. In addition, as the peak Reynolds number in coronary arteries was lower than the critical value proposed by Peacock et al. [37], the flow can be assumed to be laminar in this study.

Last but not least, the impact of errors in image processing on the simulation results is worth exploring in the future. Current clinical imaging processing techniques have difficulty in reconstructing accurate $3 \mathrm{D}$ models for small vessels (e.g., with a diameter less than $0.5 \mathrm{~mm}$ ) owing to the limitation of the imaging resolution. With the development of medical imaging techniques, the breakthrough in this area should be achievable in the near future.

\section{Abbreviations}

CAD: Coronary artery disease

CFD: Computational fluid dynamics

CT: Computed tomography

DS: Diameter stenosis

FFR: Fractional flow reserve

LAD: Left anterior descending

MI: Myocardial infarction

OSI: Oscillatory shear index

WSS: Wall shear stress. 


\section{Conflict of Interests}

The authors declare that there is no conflict of interests regarding the publication of this paper.

\section{Acknowledgments}

This research is supported by the SingHealth Foundation, Singapore, under its Translational Research Grant, and administered by the SingHealth (SHF/FG503P/2012) and by National Heart Center Singapore under its NHCS Centre Grant Seed Funding (NHCS-CGSF/2014/003), and by Biomedical Research Council Research Grant (14/1/32/24/ 002). The financial supports of NMRC/EDG/1037/2011 by the National Research Foundation, Singapore, under its Cooperative Basic Research Grant, and Duke-NUS-GCR/2013/0009, and by the Goh Cardiovascular Research Grant are gratefully acknowledged. The authors acknowledge the support of all staff at the National Heart Centre, Singapore, especially Fong Ching Teck, Max Wu Bing Huang, and Ho Pei Yi and the staff in catheter laboratory and CT departments.

\section{References}

[1] T. Heitzer, T. Schlinzig, K. Krohn, T. Meinertz, and T. Münzel, "Endothelial dysfunction, oxidative stress, and risk of cardiovascular events in patients with coronary artery disease," Circulation, vol. 104, no. 22, pp. 2673-2678, 2001.

[2] W. B. Meijboom, C. A. G. Van Mieghem, N. van Pelt et al., "Comprehensive assessment of coronary artery stenoses: computed tomography coronary angiography versus conventional coronary angiography and correlation with fractional flow reserve in patients with stable angina," Journal of the American College of Cardiology, vol. 52, no. 8, pp. 636-643, 2008.

[3] N. H. J. Pijls, J. A. M. Van Son, R. L. Kirkeeide, B. De Bruyne, and K. L. Gould, "Experimental basis of determining maximum coronary, myocardial, and collateral blood flow by pressure measurements for assessing functional stenosis severity before and after percutaneous transluminal coronary angioplasty," Circulation, vol. 87, no. 4, pp. 1354-1367, 1993.

[4] N. P. Johnson, R. L. Kirkeeide, and K. L. Gould, "Is discordance of coronary flow reserve and fractional flow reserve due to methodology or clinically relevant coronary pathophysiology?" Cardiovascular Imaging, vol. 5, no. 2, pp. 193-202, 2012.

[5] P. A. L. Tonino, B. De Bruyne, N. H. J. Pijls et al., "Fractional flow reserve versus angiography for guiding percutaneous coronary intervention," The New England Journal of Medicine, vol. 360, no. 3, pp. 213-224, 2009.

[6] N. H. J. Pijls and J.-W. E. M. Sels, "Functional measurement of coronary stenosis," Journal of the American College of Cardiology, vol. 59, no. 12, pp. 1045-1057, 2012.

[7] J. M. Zhang, L. Zhong, B. Su et al., "Perspective on CFD studies of coronary artery disease lesions and hemodynamicsa review," International Journal for Numerical Methods in Biomedical Engineering, 2014.

[8] U. Olgac, D. Poulikakos, S. C. Saur, H. Alkadhi, and V. Kurtcuoglu, "Patient-specific three-dimensional simulation of LDL accumulation in a human left coronary artery in its healthy and atherosclerotic states," American Journal of PhysiologyHeart and Circulatory Physiology, vol. 296, no. 6, pp. H1969H1982, 2009.
[9] E. Wellnhofer, J. Osman, U. Kertzscher, K. Affeld, E. Fleck, and L. Goubergrits, "Flow simulation studies in coronary arteriesImpact of side-branches," Atherosclerosis, vol. 213, no. 2, pp. 475481,2010

[10] T. Chaichana, Z. Sun, and J. Jewkes, "Computation of hemodynamics in the left coronary artery with variable angulations," Journal of Biomechanics, vol. 44, no. 10, pp. 1869-1878, 2011.

[11] R. Torii, N. B. Wood, N. Hadjiloizou et al., "Stress phase angle depicts differences in coronary artery hemodynamics due to changes in flow and geometry after percutaneous coronary intervention," American Journal of Physiology-Heart and Circulatory Physiology, vol. 296, no. 3, pp. H765-H776, 2009.

[12] H. Samady, P. Eshtehardi, M. C. McDaniel et al., "Coronary artery wall shear stress is associated with progression and transformation of atherosclerotic plaque and arterial remodeling in patients with coronary artery disease," Circulation, vol. 124, no. 7, pp. 779-788, 2011.

[13] I. E. Vignon-Clementel, C. A. Figueroa, K. E. Jansen, and C. A. Taylor, "Outflow boundary conditions for three-dimensional finite element modeling of blood flow and pressure in arteries," Computer Methods in Applied Mechanics and Engineering, vol. 195, no. 29-32, pp. 3776-3796, 2006.

[14] H. J. Kim, I. E. Vignon-Clementel, C. A. Figueroa et al., "On coupling a lumped parameter heart model and a threedimensional finite element aorta model," Annals of Biomedical Engineering, vol. 37, no. 11, pp. 2153-2169, 2009.

[15] H. J. Kim, I. E. Vignon-Clementel, J. S. Coogan, C. A. Figueroa, K. E. Jansen, and C. A. Taylor, "Patient-specific modeling of blood flow and pressure in human coronary arteries," Annals of Biomedical Engineering, vol. 38, no. 10, pp. 3195-3209, 2010.

[16] D. G. Katritsis, A. Theodorakakos, I. Pantos et al., "Vortex formation and recirculation zones in left anterior descending artery stenoses: computational fluid dynamics analysis," Physics in Medicine and Biology, vol. 55, no. 5, pp. 1395-1411, 2010.

[17] P. H. Stone, S. Saito, S. Takahashi et al., "Prediction of progression of coronary artery disease and clinical outcomes using vascular profiling of endothelial shear stress and arterial plaque characteristics: the PREDICTION study," Circulation, vol. 126, no. 2, pp. 172-181, 2012.

[18] J. Knight, U. Olgac, S. C. Saur et al., "Choosing the optimal wall shear parameter for the prediction of plaque location-a patient-specific computational study in human right coronary arteries," Atherosclerosis, vol. 211, no. 2, pp. 445-450, 2010.

[19] B.-K. Koo, A. Erglis, J.-H. Doh et al., "Diagnosis of ischemiacausing coronary stenoses by noninvasive fractional flow reserve computed from coronary computed tomographic angiograms: results from the prospective multicenter DISCOVER-FLOW (Diagnosis of Ischemia-Causing Stenoses Obtained Via Noninvasive Fractional Flow Reserve) study," Journal of the American College of Cardiology, vol. 58, no. 19, pp. 1989-1997, 2011.

[20] J. K. Min, B.-K. Koo, A. Erglis et al., "Usefulness of noninvasive fractional flow reserve computed from coronary computed tomographic angiograms for intermediate stenoses confirmed by quantitative coronary angiography," American Journal of Cardiology, vol. 110, no. 7, pp. 971-976, 2012.

[21] J. K. Min, J. Leipsic, M. J. Pencina et al., "Diagnostic accuracy of fractional flow reserve from anatomic CT angiography," Journal of the American Medical Association, vol. 308, no. 12, pp. 12371245, 2012.

[22] C. A. Taylor, T. A. Fonte, and J. K. Min, "Computational fluid dynamics applied to cardiac computed tomography for 
noninvasive quantification of fractional flow reserve," Journal of American College of Cardiology, vol. 61, no. 22, pp. 2233-2241, 2013.

[23] S. Sen, J. Escaned, I. S. Malik et al., "Development and validation of a new adenosine-independent index of stenosis severity from coronary waveintensity analysis: results of the ADVISE (ADenosine Vasodilator Independent Stenosis Evaluation) study," Journal of the American College of Cardiology, vol. 59, no. 15, pp. 1392-1402, 2012.

[24] R. F. Wilson, M. L. Marcus, and C. W. White, "Prediction of the physiologic significance of coronary arterial lesions by quantitative lesion geometry in patients with limited coronary artery disease," Circulation, vol. 75, no. 4, pp. 723-732, 1987.

[25] Y. Huo, T. Wischgol, and G. S. Kassab, "Flow patterns in threedimensional porcine epicardial coronary arterial tree," American Journal of Physiology-Heart and Circulatory Physiology, vol. 293, no. 5, pp. H2959-H2970, 2007.

[26] J.-M. Zhang, L. P. Chua, D. N. Ghista, S. C. M. Yu, and Y. S. Tan, "Numerical investigation and identification of susceptible sites of atherosclerotic lesion formation in a complete coronary artery bypass model," Medical and Biological Engineering and Computing, vol. 46, no. 7, pp. 689-699, 2008.

[27] Y. Zhou, G. S. Kassab, and S. Molloi, "On the design of the coronary arterial tree: a generalization of Murray's law," Physics in Medicine and Biology, vol. 44, no. 12, pp. 2929-2945, 1999.

[28] W. Aarnoudse, W. F. Fearon, G. Manoharan et al., "Epicardial stenosis severity does not affect minimal microcirculatory resistance," Circulation, vol. 110, no. 15, pp. 2137-2142, 2004.

[29] K. L. Gould, R. Kirkeeide, and N. P. Johnson, "Coronary branch steal experimental validation and clinical implications of interacting stenosis in branching coronary arteries," Circulation: Cardiovascular Imaging, vol. 3, no. 6, pp. 701-709, 2010.

[30] O. F. Donati, P. Stolzmann, L. Desbiolles et al., "Coronary artery disease: which degree of coronary artery stenosis is indicative of ischemia?" European Journal of Radiology, vol. 80, no. 1, pp. 120-126, 2011.

[31] S. Jost, J. W. Deckers, P. Nikutta et al., "Progression of coronary artery disease is dependent on anatomic location and diameter," Journal of the American College of Cardiology, vol. 21, no. 6, pp. 1339-1346, 1993.

[32] J. M. Zhang, T. Luo, Y. Huo et al., "Area stenosis associated with non-invasive fractional flow reserve obtained from Coronary CT Images," in Proceedings of the 35th Annual International Conference of the IEEE Engineering in Medicine and Biology Society (EMBC '13), pp. 3865-3868, 2013.

[33] L. Zhong, B. Su, J. M. Zhang et al., "Effects of stenosis on the porcine left anterior descending arterial tree," in Proceedings of the 35th Annual International Conference of the IEEE Engineering in Medicine and Biology Society (EMBC '13), pp. 3869-3872, 2013.

[34] J. E. Davies, Z. I. Whinnett, D. P. Francis et al., "Evidence of a dominant backward-propagating "suction" wave responsible for diastolic coronary filling in humans, attenuated in left ventricular hypertrophy," Circulation, vol. 113, no. 14, pp. 17681778, 2006.

[35] S. B. Bender, M. J. Van Houwelingen, D. Merkus, D. J. Duncker, and M. Harold Laughlin, "Quantitative analysis of exerciseinduced enhancement of early- and late-systolic retrograde coronary blood flow," Journal of Applied Physiology, vol. 108, no. 3, pp. 507-514, 2010.

[36] D. Zeng, E. Boutsianis, M. Ammann, K. Boomsma, S. Wildermuth, and D. Poulikakos, "A study on the compliance of a right coronary artery and its impact on wall shear stress," Journal of Biomechanical Engineering, vol. 130, no. 4, Article ID 041014, 11 pages, 2008.

[37] J. Peacock, T. Jones, C. Tock, and R. Lutz, "The onset of turbulence in physiological pulsatile flow in a straight tube," Experiments in Fluids, vol. 24, no. 1, pp. 1-9, 1998. 

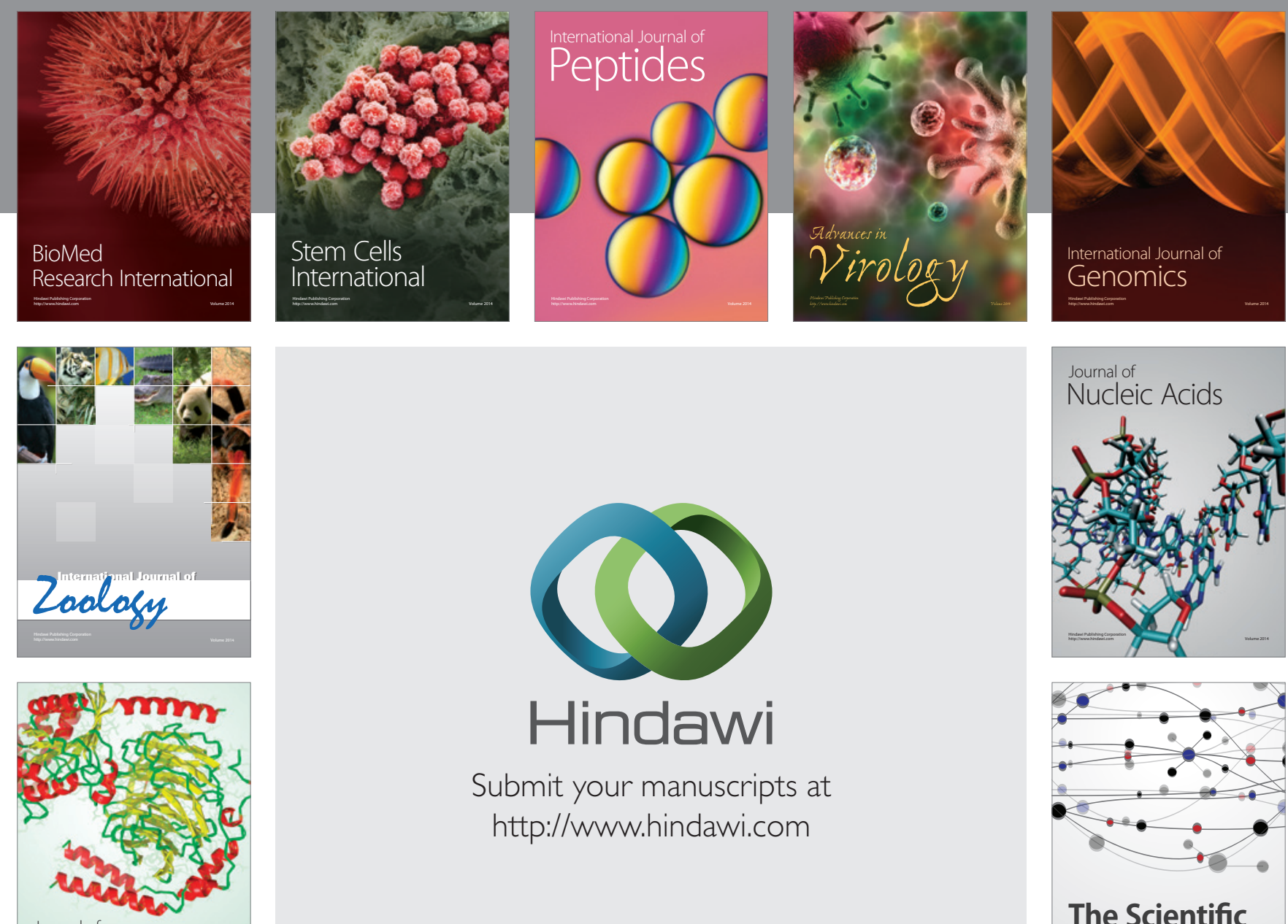

Submit your manuscripts at

http://www.hindawi.com

Journal of
Signal Transduction
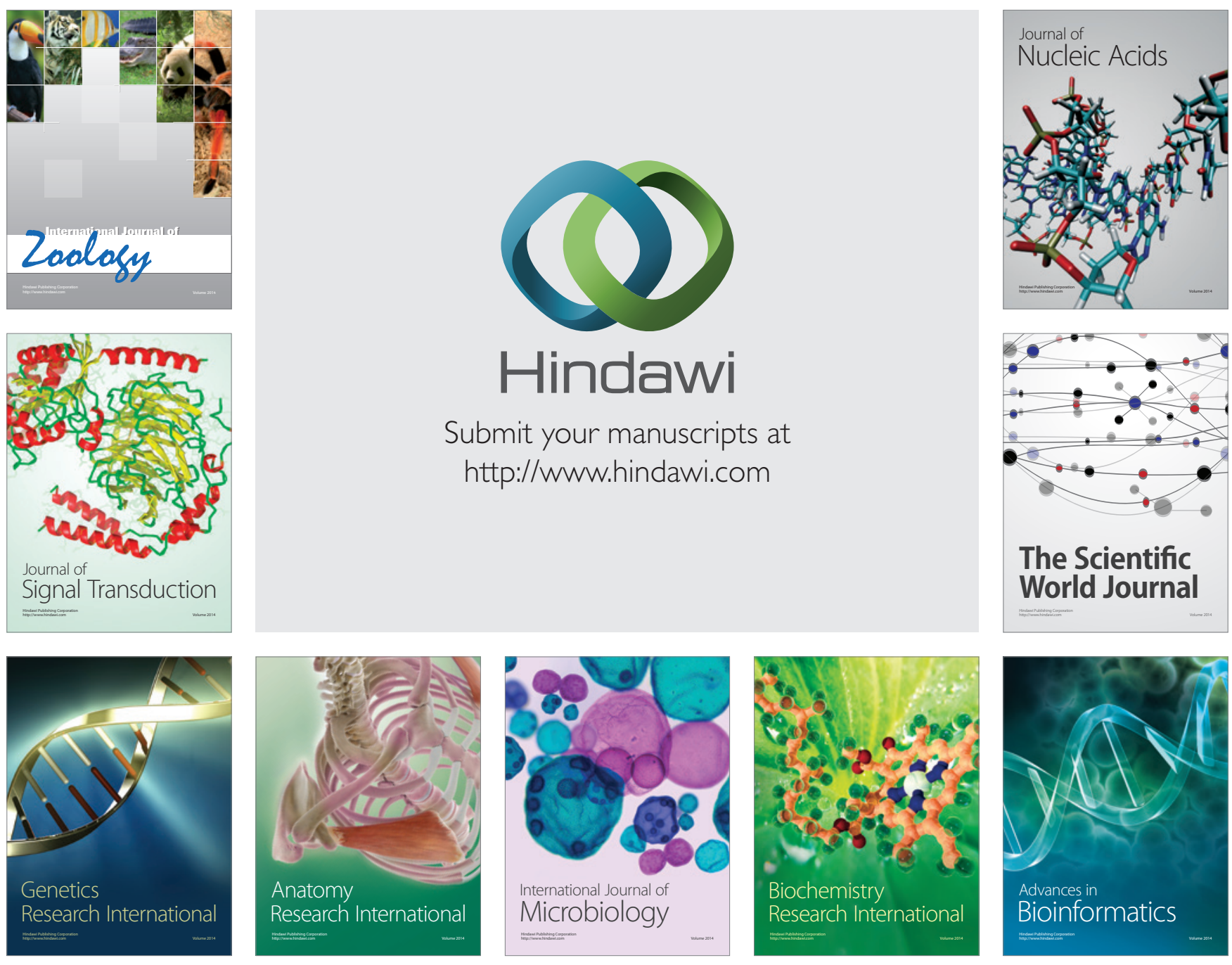

The Scientific World Journal
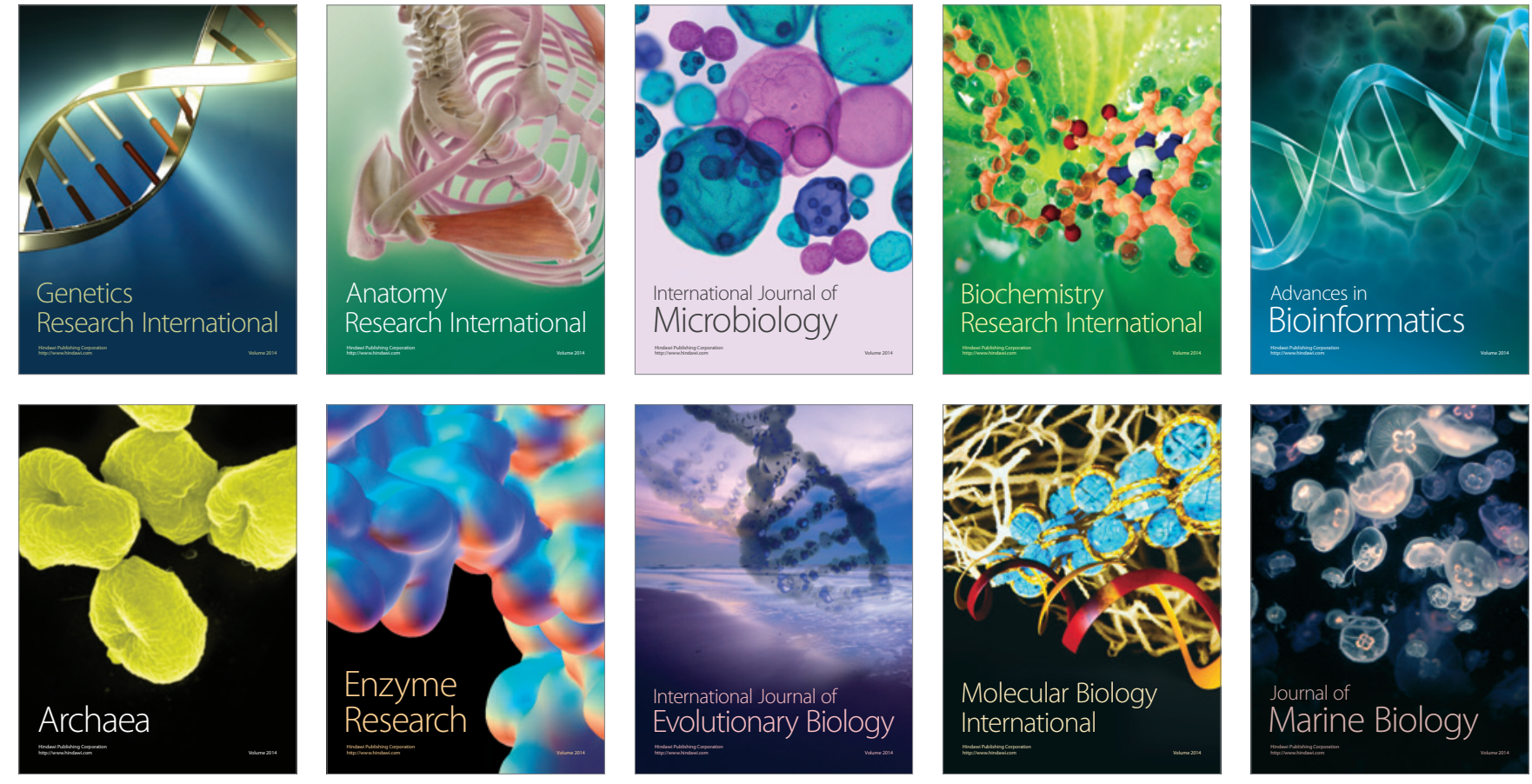